$\mathbf{m} / \mathbf{S}$

médecine/sciences $1986 ; 2: 356-7$

\title{
MÉTABOLISME HÉPATIQUE DES MÉDICAMENTS ET LÉSIONS HÉPATIOUES MÉDICAMENTEUSES
}

\section{Jean-Pierre \\ Benhamou}

Professeur à la faculté de médecine XavierBichat.

Chef du service d'hépatologie de l'hôpital Beaujon.

\section{ADRESSE}

J.-P. Benhamou : service d'hćpatologie et unité de recherches de physiopathologie hépatique (Inserm U 24), hôpital Beaujon, 92 I 18 Clichy cedex.

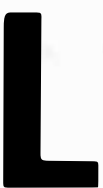

es lésions du foie induites par les médicaments représentent actuellement un des aspects les plus fascinants de l'hépatologie, du fait de leur diversité, de leur fréquence, de leur mécanisme, de la difficulté de leur reconnaissance, et de la difficulté de leur prédiction.

Les médicaments peuvent déterminer presque tous les types de lésions hépatiques. Les plus fréquentes sont les hépatites cytolytiques aiguës, caractérisées par une nécrose des hépatocytes. Les hépatites aiguës cholestatiques, moins fréquentes, sont caractérisées par une cholestase due à un dysfonctionnement des hépatocytes et/ou à des lésions des petites voies biliaires intrahépatiques; elles peuvent être suivies d'une cholestase pouvant durer quelques mois ou quelques années [I]. Les autres lésions hépatiques, indiquées dañs le tableau $I$, sont d'observation plus rare.

La fréquence des lésions hépatiques médicamenteuses dépend de la consommation globale des médicaments. Leur fréquence est relativement élevée dans les pays industrialisés, mais reste faible dans les pays en voie de développement. D'après mon expérience à l'hôpital Beaujon, les médicaments sont responsables d'environ $5 \%$ des hépatites aiguës chez des sujets de moins de 40 ans, de $30 \%$ des hépatites aiguës chez des sujets de 40 à 60 ans, et de $60 \%$ des hépatites aiguës chez des sujets de plus de 60 ans. Cette fréquence chez le sujet âgé est due à la consommation accrue de médicaments, mais, peut-être aussi, à un métabolisme différent du médicament.

Le mécanisme des lésions hépatiques médicamenteuses est mal connu. Dans les hépatites aiguës cytolytiques, un rôle déterminant semble être joué par les métabolites réactifs des médicaments. Dans la biotransformation des médicaments par les cytochromes $\mathrm{P}-450$, le médicament peut être transformé par oxydation en un ou plusieurs métabolites réactifs. S'ils s'unissent à des macromolécules indispensables au fonctionnement des hépatocytes, il en résulte une lésion hépatique. Les hépatites liées à ce type d'hépatotoxicité ont les caractéristiques suivantes : $(a)$ les lésions prédominent dans la région centrolobulaire, où la concentration de certains cytochromes $\mathrm{P}-450$ est la plus élevée; $(b)$ le risque de lésions hépatiques est augmenté par une induction enzymatique (qui entraîne la formation d'une quantité accrue de métabolites réactifs). Un exemple typique.de ce mécanisme est représenté par l'hépatite due à l'administration simultanée d'isoniazide et de

\section{RÉFÉRENCES}

I. Larrey D, Pessayre D, Duhamel $\mathrm{G}$, et al. Prolonged cholestasis af ter ajmaline-induced acute hepatitis. 7 Hepatol 1986; $2: 81-7$.

2. Pessayre D, Bentata M, Degott C, et al. Isoniazid-rifampin fulminant hepatitis. A possible consequence of the cnhancement of isoniazid hepatotoxicity by enzyme induction. Gastroenterology 1977; $72: 284-9$.

3. Pessayrc D, de Saint Louvent $P$, Degott C, Bcrnuau J, Rueff B, Benhamou JP. Iproclozide fulminant hepatitis. Possible role of enzyme induction. Gastroentero$\log y 1978 ; 75: 492-6$.

4. Funck-Brentano C, Pessayre D, Benhamou JP. Hépatites ducs à divers dérivés de l'érythromycine. Gastroenterol Clin Biol 1983; 7 : 362-9.

5. Stricker BHC, Spoelstra P Drug-induced hepatic injury. $A$ comprehensive survey of the literature on adverse drug reactions up to January 1985. Amsterdam : Elsevier, $1985: 314 \mathrm{p}$. 
rifampicine [2]: un métabolite réactif est formé à partir de l'isoniazide; la rifampicine est un inducteur enzymatique; de ce fait, les sujets qui reçoivent simultanément de la rifampicine et de l'isoniazide forment, à partir de ce dernier médicament, plus de métabolites réactifs et sont soumis à un risque plus élevé d'hépatite. Dans cet exemple, le médicament hépatotoxique et l'inducteur enzymatique étaient administrés simultanément. Dans certains cas, le médicament hépatotoxique, l'iproclozide, a été administré isolément pendant une longue période sans entraîner de lésions hépatiques. Puis, un barbiturique, qui possède des propriétés d'induction enzymatique, a été prescrit et, favorisant la formation d'une quantité accrue de métabolites réactifs à partir de l'iproclozide, a entraîné une hépatite fulminante. Dans de tels cas, on aurait pu penser à tort à une hépatite due au barbiturique [3].

Les métabolites réactifs peuvent être hépatotoxiques par un deuxième mécanisme. Le complexe formé par la macromolécule et les métabolites réactifs peut se comporter comme un allergène et déterminer une réponse immunitaire dirigée contre les hépatocytes contenant l'allergène. Les hépatites liées à ce deuxième type d'hépatotoxicité ont les caractéristiques suivantes : (a) l'hépatite s'accompagne souvent de manifestations de type allergique, en particulier une éruption cutanée et une éosinophilie sanguine; (b) la réadministration du médicament provoque une récidive dans un délai beaucoup plus court.

Les deux mécanismes qui viennent d'être décrits ne s'excluent pas. Ainsi, l'administration d'estolate d'érythromycine entraîne chez I $5 \%$ des sujets traités une élévation modérée des transaminases: ce phénomène, relativement fréquent, pourrait être de mécanisme toxique. A l'opposé, l'hépatite avec ictère est rare; elle s'accompagne fréquemment de manifestations d'hypersensibilité et récidive précocement après une deuxième administration [4].

La reconnaissance des lésions hépa- tiques médicamenteuses est souvent difficile. Je me limiterai ici aux seuls problèmes soulevés par le diagnostic des hépatites aiguës médicamenteuses. Leur diagnostic a bénéficié de la meilleure reconnaissance des hépatites aiguës virales. L'absence d'anticorps de type IgM contre le virus $A$ permet d'exclure une hépatite virale $A$. De même, l'absence d'antigène HBs et d'anticorps anti$\mathrm{HBc}$ de type IgM permet d'exclure une hépatite virale $B$. A partir du moment où les deux principales hépatites virales sont exclues, il convient d'envisager soigneusement le diagnostic d'hépatite médicamenteuse, particulièrement chez les sujets qui ont plus de 40 ans. Il conviendra de rechercher attentivement la prise d'un médicament réputé hépatotoxique dans les jours, les semaines, ou les mois qui ont précédé l'hépatite. Pour savoir si un médicament est connu comme pouvant déterminer une lésion hépatique, le clinicien est beaucoup aidé par l'ouvrage de Stricker et Spoelstra [5] dans lequel est répertoriée toute l'information sur ce point. La preuve la plus certaine de l'étiologie médicamenteuse d'une hépatite aiguë est la récidive après réadministration du médicament. Toutef ois, cette réadministration doit être déconseillée, car elle peut déterminer une récidive sévère. Mais la réadministration peut être involontaire, la première hépatite n'ayant pas été rapportée à son étiologie médicamenteuse.

Comment prédire la survenue d'une hépatite médicamenteuse? Le risque d'hépatite chez les malades recevant un médicament donné est difficilement prévisible avant la commercialisation du produit. Dans la plupart des cas, la fréquence de l'hépatite médicamenteuse, du moins dans sa forme symptomatique, est de l'ordre de i pour ıoo à I pour 10000 . Il est donc indispensable, au début de la commercialisation d'un médicament, au moment où il va être administré à des milliers, voire à des centaines de milliers de personnes, d'être particulièrement vigilant.

Il convient d'êtrè spécialement attentif dans les situations sui- vantes : $(a)$ lorsque l'administration de fortes doses du médicament détermine chez l'animal des lésions hépatiques; $(b)$ lorsque le médicament forme chez l'animal de grandes quantités de métabolites réactifs; (c) lorsque les études effectuées avant la commercialisation ont permis de constater une élévation asymptomatique des transaminases chez un certain nombre de sujets recevant le médicament. Toutefois, la prédiction de l'hépatotoxicité reste difficile : le métabolisme du médicament peut être différent chez l'animal et chez l'homme et, de ce fait, n'être pas hépatotoxique chez le premier et l'être chez le second. Il arrive qu'un médicament élève assez souvent les transaminases chez l'homme et ne détermine jamais d'hépatite symptomatique : ainsi, les fortes doses d'aspirine entraînent une élévation asymptomatique des transaminases, sans déterminer d'hépatite symptomatique

\begin{tabular}{l} 
Tableau I \\
\multicolumn{1}{|c|}{ LÉSIONS HÉPATIQUES } \\
MÉDICAMENTEUSES \\
\hline Hépatite \\
- aiguë \\
cytolytique \\
cholestatique \\
mixte \\
- chronique \\
Granulomatose \\
Thésaurismose \\
- triglycérides (stéatose) \\
$\quad$ stéatose macrovésiculaire \\
stéatose microvésiculaire \\
- phospholipides \\
- hypervitaminose A \\
- fer (hémosidérose et hémochro- \\
matose) \\
Lésions vasculaires et sinu- \\
soïdales \\
- thrombose de la veine porte \\
- thrombose de l'artère hépatique \\
- infarctus du foie \\
- dilatation sinusoïdale \\
- péliose \\
- maladie veino-occlusive \\
- thrombose des veines sus-hépa- \\
tiques \\
Tumeurs hépatiques \\
- adénome hépatocellulaire \\
- carcinome hépatocellulaire \\
- carcinome cholangiocellulaire \\
- angiosarcome \\
Cholangite sclérosante \\
\hline
\end{tabular}

